\title{
Evaluation of the running parameters of the polar cranes installed in the NPP reactor compartments during their control assembly
}

\author{
Yuri I. Pimshin ${ }^{1, *}$, Yuri V. Zayarov ${ }^{1}$, and Ivan Yu. Pimshin ${ }^{2}$ \\ ${ }^{1}$ Volgodonsk Engineering Technical Institute the branch of National Research Nuclear University \\ "MEPhI" \\ ${ }^{2}$ Don State Technical University (DSTU)
}

\begin{abstract}
The paper considers the basic principles of motion along the polar cranes circumference. The geometric parameters of the mutual turn of the crane running wheels are shown to be the critical features in the crane motion along the circumference. Noncompliance of the turn determines the transverse slippage of the crane running wheels, while the wheel shape affects only the elastic longitudinal slip. The consequence of slide and slippage is the railhead actuation on one side and the loss of the road stability when the crane moves. Stability of the road-holding rigidity ensures achievement of targeting accuracy of the load-gripping device. These parameters are the main operational characteristics which should be normalized and should be minimal in absolute terms.
\end{abstract}

\section{Introduction}

At modern nuclear power plants under construction at NPP-2006 and WWER-TOI design the polar cranes is the primary transport facility for the main lifting and transport operations in the hermetic volume when installing the equipment of the primary circuit (reactor shell, steam generators, main circulation pumps, pipelines) and refueling (Fig. 1). At the operation stage, the crane is used for lifting and transporting covers with fresh fuel from the transport corridor into the storage pool saddle and for removing the spent fuel containers from it and accompanying the mechanic work on replacing the equipment in the reactor compartment during the current preventive maintenance. Table 1 shows the main characteristics of the crane. One of the main features of the polar cranes is their movement along the circle. The organization of this motion is carried out by certain geometric conditions realized in the running part of the crane (Fig. 2). The main operational characteristics of the crane depend on the accuracy of the following geometric conditions:

- guidance accuracy;

- longitudinal stability;

- longitudinal and cross slip.

\footnotetext{
* Corresponding author: YIPimshin@mephi.ru
} 
In the Russian Federation these types of cranes are manufactured at the "Tyazhmash" plant in Syzran and at the JSC Baltkran in Kaliningrad.

\section{The main proposals of the study with justification of the obtained scientific results}

The modern design of the polar crane is developed by the design department of the "Tyazhmash" plant. The project has a non-skid mounted installation in the small balancers of the running wheels. This determines the stringent requirements for the accuracy of the running wheel manufacturing, their installation in small and then in main balancers. Manufacturing errors can not be corrected such as in the running gear with the wheel installation in the balancers with the help of rotors. In this design production errors can be eliminated only by the new manufacture of parts. In this regard a very careful monitoring of the crane manufacturing should be carried out with its control assembly at the plant.

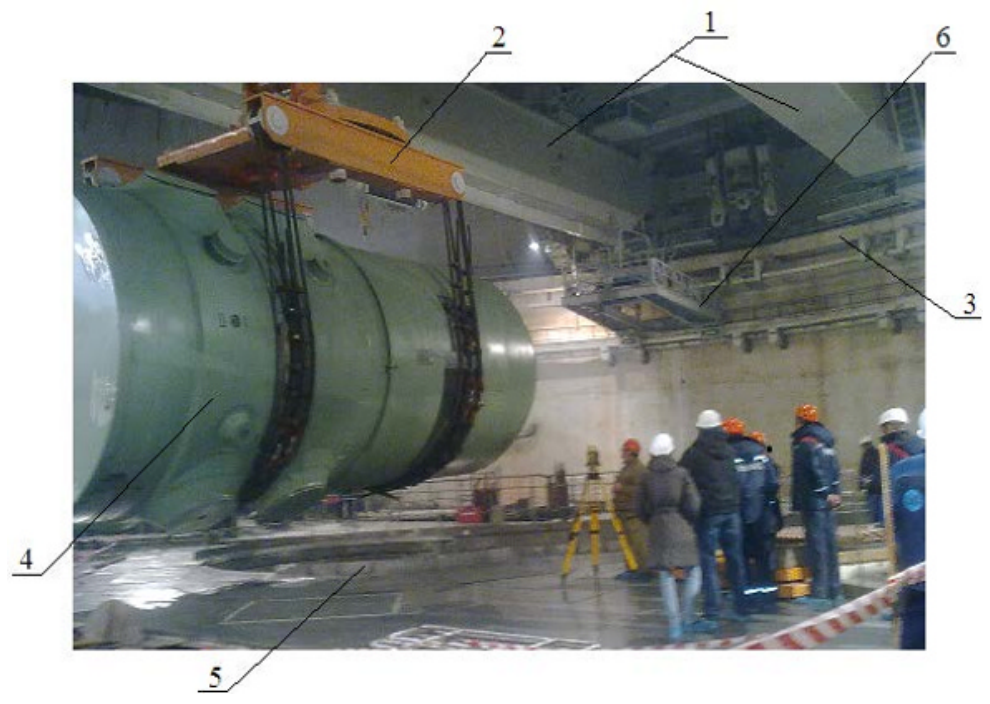

Fig. 1. Transportation of the reactor vessel to the reactor pool for its installation: 1- longitudinal beam of polar cranes; 2 - traverse mounted on the main crane; 3 - crane beam of polar crane; 4- reactor vessel; 5- reactor pool; 6- cantilever polar crane.

Table 1. Polar Crane Parameters

\begin{tabular}{|c|l|c|}
\hline № $\Pi / \Pi$ & \multicolumn{1}{|c|}{ Parameter Type } & Parameter Value \\
\hline 1 & Crane span: & $43000 \mathrm{~mm}$ \\
\hline 1.1 & WWER-1000 NPP reactor & $41500 \mathrm{~mm}$ \\
\hline 1.2 & NPP-2006 and WWER-TOI & $500-600$ and more t. \\
\hline 2 & Crane weight & till $400 \mathrm{t}$. \\
\hline 3 & Capacity & $\pm 7 \mathrm{~mm}$ \\
\hline 4 & Target accuracy & $\pm 2.5 \mathrm{~mm}$ \\
\hline 5 & Running wheel setting accuracy: & $\pm 5 \mathrm{~mm}$ \\
\hline 5.1 & at a given radius & $\pm 1 \mathrm{~mm}$ \\
\hline 5.2 & at a given diameter & \\
\hline 6 & Permissible turn of the wheel relative to the & \\
\hline
\end{tabular}


There is a possibility to correct identified inadmissible inaccuracies according to the results of this control. It is much more difficult and more costly to make restoration work in the construction object.

Determining the crane bridge parameters and the parameters of its running gear are made during the testing of the polar crane after its assembly.

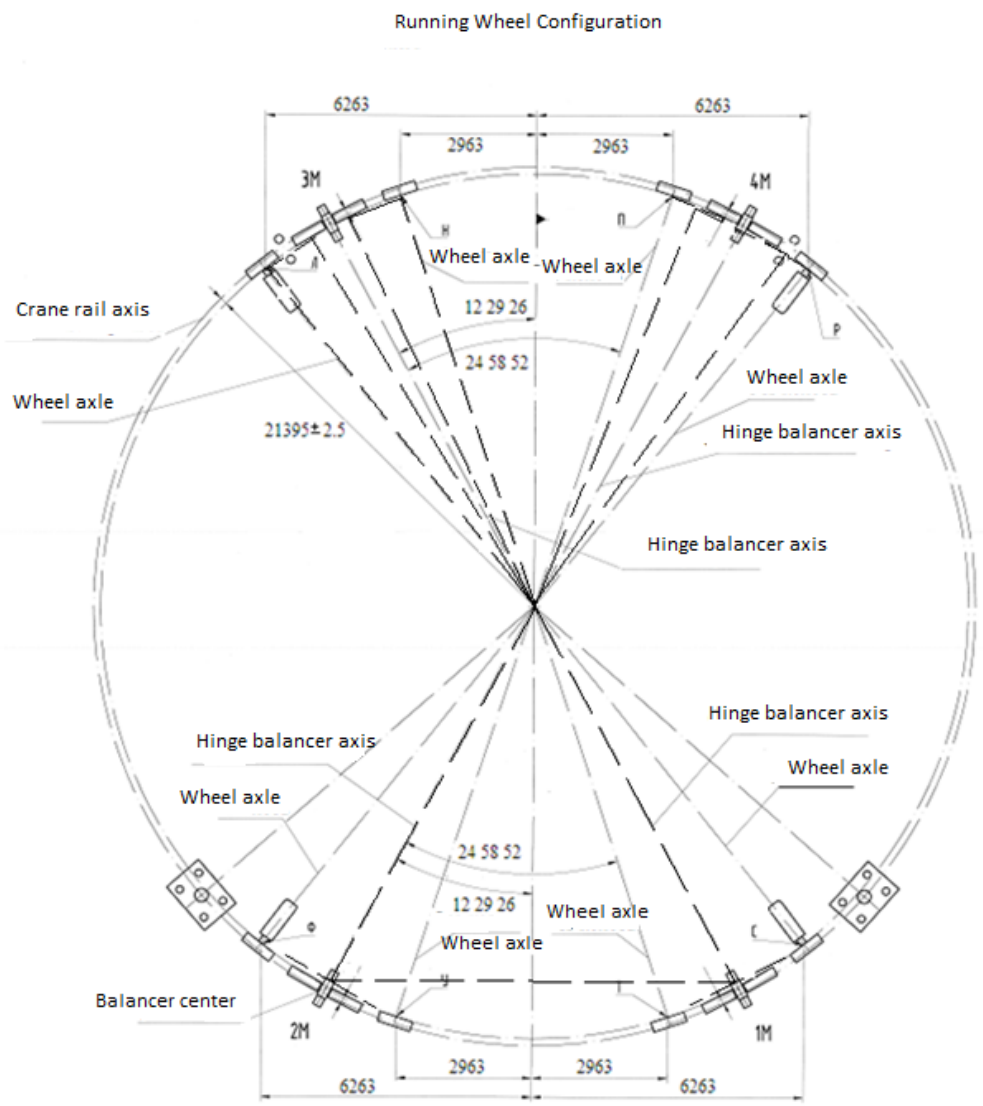

Fig. 2. Crane running gear configuration.

\section{Geometric Parameter Checking of the Polar Crane Bridge}

When checking the geometric parameters of the polar crane bridge, the work is carried out to determine the sides and diagonals of the rectangles formed by the points $\mathrm{K}$ and $\mathrm{F}$ (Fig. 3). The results of the completed work are given in the logbooks. Besides the bridge main beams is determined. Results of measurements are given in tabular and graphical forms.

\section{Geometric Characteristics Monitoring of the Polar Crane Running Gear}

When checking the geometric parameters of the polar crane running gear, work is carried out to determine the wheel geometric parameters, their actual radii (Fig. 4, Table 2) and the installation parameters in the crane running system (Fig. 2), their mutual position parameters. According to the control results the longitudinal and transverse sliding parameters are calculated: 


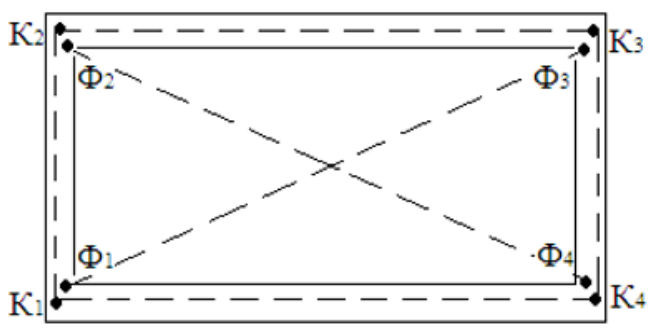

Fig. 3. Scheme of the crane bridge monitored dimensions

- Sliding is a continuous process of interaction between the wheel and the rail where the length of the traversed path counted on the railhead is not equal to the length of the track counted along the wheel generatrix.

- Slippage is the momentary change process in the mutual position of the wheel and rail with the unloading of accumulated stresses in the crane metal structures.

In this case the sliding can be formed both in the longitudinal direction (coinciding with the motion direction) and in the transverse direction (perpendicular to the motion direction).

Slippage is realized only in the transverse direction. The consequence of sliding and slippage is the actuation of the railhead on one side and the loss of the road stability when the crane moves.

These parameters are the main operational characteristics which should be normalized and should be minimal in absolute terms.

Transverse sliding. When the crane moves in the case when $R_{\Pi / \pi}$ is not equal to $R$ (wheel rolling) then the wheel tends to move along its circle corresponding to its geometric parameters. However, being in the crane wheel running system, the given wheel remains on the circumference of the crane track. Thus, there is a constant sliding of the wheel in the radial direction by $\Delta_{\mathrm{x}}$ value when rolling the wheel at each complete turn $\mathrm{C}_{\mathrm{K}}$. The coefficient of relative cross sliding is calculated by the formula

$$
K_{\Delta x}=\Delta_{x} / C_{\kappa} .
$$

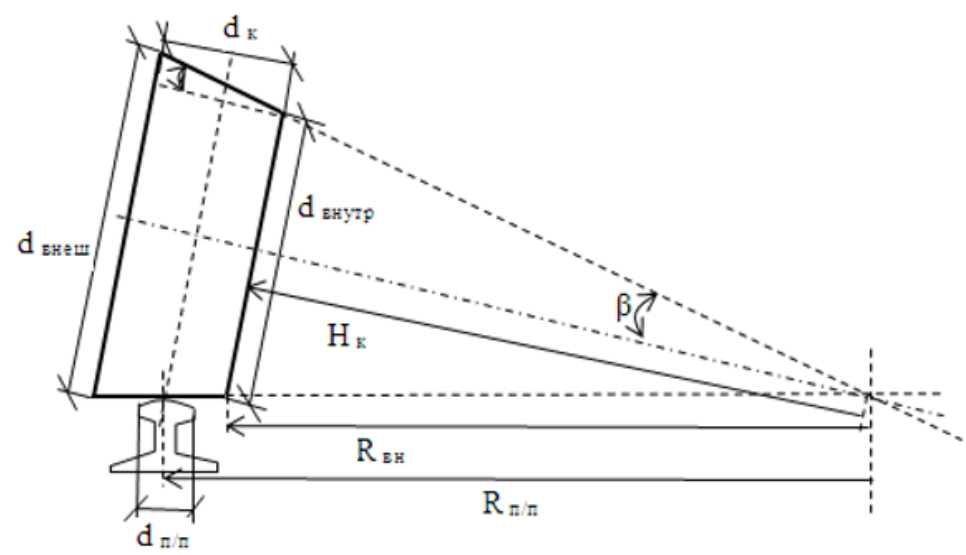

Fig. 4. Scheme of theoretical geometric parameters of the wheel

Table 2. Theoretical geometric parameters of the wheel

\begin{tabular}{|c|c|c|c|c|c|c|c|}
\hline $\mathrm{d}_{\text {ext }}$ & $\mathrm{d}_{\text {in }}$ & $\mathrm{d}_{\mathrm{\kappa}}$ & $\mathrm{R}_{\text {in }}$ & $\mathrm{H}_{\mathrm{\kappa}}$ & $\beta$ & $\mathrm{R}_{\text {п/п }}$ & $\mathrm{d}_{\text {п/п }}$ \\
\hline 745,2 & 738,0 & 210 & 21395 & 21391,82 & $59^{\prime} 17,63^{\prime \prime}$ & 21500 & 140 \\
\hline
\end{tabular}


Longitudinal sliding. When installing the wheel on the rail in the area of contact, a socalled "contact spot" is formed. Its shape depends on the shape of the generatrix wheel and the shape of the railhead upper surface. In this case if the rail in the plan has a radial shape then rolling the wheel along this rail in the contact spot is the process of longitudinal sliding $\Delta_{c}$. If the wheel is conical and $\mathrm{R}_{\Pi / \pi}=\mathrm{R}$ (wheel rolling) then $\Delta_{\mathrm{c}}=0$. In any other case the longitudinal sliding process $\Delta_{c}$ is formed in the contact spot. The coefficient of relative longitudinal sliding is calculated by the formula

$$
K_{\Delta c}=\Delta_{c} / C_{K} .
$$

The most important characteristics of the crane running gear geometric parameters are the parameters of the relative position of the running wheels in small balancers. The mutual arrangement of the wheels determines the crane movement along the circumference. At the same time if the conical wheels with a motion radius corresponding to the crane track radius are used in the crane running gear and the cone tops of these wheels are aligned in a single center the forces of mutual influence are zero $f_{\kappa}=0$. The crane moves along the calculated circle with a radius equal to $R_{n / n}$ without any efforts in the small balancers. In any other cases, for example, when the radius of the conical wheels is not equal to the calculated $R_{n / n}$ or their vertices are not aligned in a single center, there are forces $f_{\kappa}$ of the mutual influence of the wheels in the small balancers that characterize the conditions of motion along the circle $\left(R_{i}\right.$ is not equal to $\left.R_{n / n}\right)$, the geometric parameters of which are determined by the values of these forces. Without considering the theoretical basis of these provisions in detail, we note that a virtual conical wheel can replace any pair of wheels combined in small balancers. The parameters of this wheel are similar to the parameters of real cylindrical wheels with one exception. There is no concept of longitudinal sliding for the virtual wheel. The virtual wheel forms only a transverse sliding in the crane running system. Besides, the parameters of the virtual wheels for the main balancers and for the crane side (two balancers of each side of the crane) are constructed and calculated in the system of crane running wheels.

\section{Summary}

According to all calculated parameters, we can calculate the values of longitudinal and transverse sliding, longitudinal stability; guidance accuracy. That is, a customer has a full idea of the crane running characteristics and can either sign the acceptance certificate or require correction of the revealed manufacturing errors during crane testing assembly. As a result, the implementation of these proposals improves the quality of the polar cranes installed in the NPP reactor compartments.

\section{References}

1. Pimshin Yu.I., Pimshin I.Yu., Naugolnov V.A. Diagnosing Method of Geometric Parameters of Bridge Crane Running Gear: Patent No. 2382347 G01M 17/06. Published 02/20/2010, Bulletin No. 5.

2. Pimshin Yu.I., Pimshin I.Yu., Naugolnov V.A. Diagnosing Method of Geometric Parameters of Radial Bridge Crane Running Gear: Patent No. 2384831 G01M 17/06. Published 20.03.2010, Bulletin No. 8.

3. Pimshin Yu.I., Naugolnov V.A., Pimshin I.Yu. Diagnosing Method of Geometric Parameters of Polar Crane Running Gear: Patent No. 2425348 G01M 17/06. Published 27.07.2011, Bulletin No. 21. 